\title{
ON OPERATOR-VALUED MONOTONE INDEPENDENCE
}

\author{
TAKAHIRO HASEBE AND HAYATO SAIGO
}

\begin{abstract}
We investigate operator-valued monotone independence, a noncommutative version of independence for conditional expectation. First we introduce operator-valued monotone cumulants to clarify the whole theory and show the moment-cumulant formula. As an application, one can obtain an easy proof of the central limit theorem for the operator-valued case. Moreover, we prove a generalization of Muraki's formula for the sum of independent random variables and a relation between generating functions of moments and cumulants.
\end{abstract}

\section{$\S 1$. Introduction}

Noncommutative probability is an algebraic generalization of (Kolmogorovian) probability theory and quantum theory. A noncommutative probability space is a pair of a $*$-algebra $\mathcal{A}$ and a state $\varphi$ on $\mathcal{A}$ (i.e., a linear functional $\varphi: \mathcal{A} \rightarrow \mathbb{C}$ with the positivity $\varphi\left(a^{*} a\right) \geq 0$ for $\left.a \in \mathcal{A}\right)$. Given a probability space $(\Omega, \mathcal{F}, P)$, we can associate a pair of a commutative $*$ algebra $L^{\infty}(\Omega, \mathcal{F}, P)$ and a state $E$, which is an expectation regarding $P$. In quantum theory, $\mathcal{A}$ is called the observable algebra. Noncommutativity of the algebra implies many important physical consequences, such as the uncertainty principle.

One of the most striking features of noncommutative probability is that the concept of independence is not unique. In addition to the usual notion of independence in probability theory (tensor independence), free, Boolean, and monotone independences were introduced in [15], [14], and [9], respectively. These four notions can be characterized by natural properties (see [8]).

Moreover, we can also consider a noncommutative version of conditional independence, replacing a state with a conditional expectation taking values

Received July 17, 2012. Revised May 27, 2013. Accepted August 19, 2013.

First published online July 22, 2014.

2010 Mathematics Subject Classification. Primary 46L53; Secondary 46L54, 13F25, 06A07. 
in a possibly noncommutative algebra. This kind of independence is called operator-valued independence. For instance, the reader is referred to [3], [13], and [16] for the free case, [7] and [11] for the Boolean case, and [10] and [12] for the monotone case.

Among the four independences, monotone independence is particularly difficult to treat since the concept of mutual independence fails to hold. More precisely, random variables $Y$ and $X$ may not be independent even if $X$ and $Y$ are independent. So we have to distinguish $(X, Y)$ and $(Y, X)$. On the other hand, monotone independence plays a crucial role in some situations. For instance, monotone independence is essential to understand the relation between a free Lévy process and a classical Markov process, as shown by Biane [2, Theorem 4.2] implicitly (Franz pointed out this relation explicitly in [4, Corollary 5.3]).

In this paper, we develop a theory on operator-valued monotone independence for multivariate random variables, generalizing results of Popa [10] for a single random variable. We extend the Muraki formula, which describes the moments of the sum of independent random variables, to the operator-valued setting.

First, we define the notion of an operator-valued version of generalized cumulants to clarify the whole theory and prove the moment-cumulant formula, following the idea of [5]. We apply this to the central limit theorem, to obtain a new expression of the limit distribution. Then we investigate generating functions of moments and cumulants. To this end, we extend the algebraic structure of the ring of multivariate formal power series, focusing on the coefficients of series. Finally, we prove the extension of the Muraki formula and a differential equation involving moments and cumulants.

\section{$\S 2$. Operator-valued monotone independence}

\subsection{Preliminary concepts}

Involutions on algebras are not essential in the scope of this paper, so we do not consider them below. In this paper, $\mathfrak{B}$ denotes a unital algebra, and $\mathcal{A}$ denotes a unital algebra containing $\mathfrak{B}$ as a subalgebra. We assume that the unit of $\mathfrak{B}$ coincides with that of $\mathcal{A}$. In this paper, algebras can be considered over any commutative field, such as $\mathbb{R}$ and $\mathbb{C}$. We say that $\mathcal{C}$ is a subalgebra of $\mathcal{A}$ over $\mathfrak{B}$ if $\mathcal{C}$ is a subalgebra of $\mathcal{A}$ and if $b c \in \mathcal{C}$ for all $b \in \mathfrak{B}$ and $c \in \mathcal{C}$. A subalgebra of $\mathcal{A}$ over $\mathfrak{B}$ may not contain the unit of $\mathcal{A}$. 
For $X_{1}, \ldots, X_{r} \in \mathcal{A}$, let $\mathfrak{B}\left\langle X_{1}, \ldots, X_{r}\right\rangle_{0}$ denote the subalgebra of $\mathcal{A}$ over $\mathfrak{B}$ consisting of finite sums of elements of $\left\{b_{1} X_{i_{1}} b_{2} \cdots X_{i_{n}} b_{n+1}: b_{i} \in\right.$ $\left.\mathfrak{B}, n \geq 1, i_{1}, \ldots, i_{n} \in\{1, \ldots, r\}\right\}$. Note that, in general, $\mathfrak{B}$ is not contained in $\mathfrak{B}\left\langle X_{1}, \ldots, X_{r}\right\rangle_{0}$.

Let $\mathfrak{D}$ be another unital algebra containing $\mathfrak{B}$ as a subalgebra. A map $f$ from $\mathcal{A}$ to $\mathfrak{D}$ is called $\mathfrak{B}$-linear if $f\left(b_{1} x b_{2}+y\right)=b_{1} f(x) b_{2}+f(y)$ for all $b_{1}, b_{2} \in \mathfrak{B}$ and $x, y \in \mathcal{A}$. A $\mathfrak{B}$-linear map $h$ is called a $\mathfrak{B}$-homomorphism if $h(x y)=h(x) h(y)$ for any $x, y \in \mathcal{A}$. A $\mathfrak{B}$-linear map $\varphi$ with values in $\mathfrak{B}$ is called a conditional expectation if $\varphi(b)=b$ for $b \in \mathfrak{B}$. From now on we assume that $\varphi$ is a conditional expectation in the above sense. A triple $(\mathcal{A}, \mathfrak{B}, \varphi)$ is called an algebraic probability space or a noncommutative probability space, as in the case $\mathfrak{B}=\mathbb{C}$.

A random variable is an element of $\mathcal{A}$, and a random vector or vectorvalued random variable is an element of $\mathcal{A}^{r}$ for an $r \geq 1$.

The concept of (joint) moments has to be generalized, since $\varphi\left(X_{i_{1}} \cdots X_{i_{n}}\right)$ $\left(1 \leq i_{1}, \ldots, i_{n} \leq r\right)$ are not sufficient to know the information on a conditional expectation.

Definition 2.1. Let $X=\left(X_{1}, \ldots, X_{r}\right)$ be a random vector, and let $i_{1}, \ldots$, $i_{n} \in\{1, \ldots, r\}$ for $n \geq 1$. The multilinear functional $\mu_{i_{1}, \ldots, i_{n}}^{X}$ defined by

$$
\mu_{i_{1}, \ldots, i_{n}}^{X}\left(b_{1}, \ldots, b_{n}\right)=\varphi\left(b_{1} X_{i_{1}} b_{2} \cdots b_{n} X_{i_{n}}\right)
$$

is called an $\left(i_{1}, \ldots, i_{n}\right)$-moment of $X$.

The monotone independence over $\mathfrak{B}$ was introduced by Skeide [12].

Definition 2.2. Let $\Lambda$ be an index set equipped with a linear order $<$. A family of subalgebras $\left(\mathcal{A}_{\lambda}\right)_{\lambda \in \Lambda}$ over $\mathfrak{B}$ is said to be monotone independent over $\mathfrak{B}$ if

$$
\varphi\left(X_{1} \cdots X_{n}\right)=\varphi\left(X_{1} \cdots X_{i-1} \varphi\left(X_{i}\right) X_{i+1} \cdots X_{n}\right)
$$

holds for any $X_{i} \in \mathcal{A}_{\lambda_{i}}$ whenever $i$ satisfies $\lambda_{i-1}<\lambda_{i}$ and $\lambda_{i}>\lambda_{i+1}$. (One of the inequalities is eliminated when $i=1$ or $i=n$.) Independence for random vectors $X_{\lambda}=\left(X_{\lambda, 1}, \ldots, X_{\lambda, k_{\lambda}}\right), \lambda \in \Lambda$ is defined by considering the subalgebras $\mathcal{A}_{\lambda}:=\mathfrak{B}\left\langle X_{\lambda, 1}, \ldots, X_{\lambda, k_{\lambda}}\right\rangle_{0}$.

Let $\Lambda$ be a linearly ordered set, and let $r \in \mathbb{N}, r \geq 1$. Random vectors $X_{\lambda}=$ $\left(X_{\lambda, 1}, \ldots, X_{\lambda, r}\right), \lambda \in \Lambda$ are said to be monotone independent, identically distributed (i.i.d.) if they are monotone independent over $\mathfrak{B}, \mu_{i_{1}, \ldots, i_{n}}^{X_{\lambda}}$ does not depend on $\lambda \in \Lambda$ for any $i_{1}, \ldots, i_{n} \in\{1, \ldots, r\}$, and $n \geq 1$. 


\subsection{Dot operation}

We introduce a dot operation, following [5].

Definition 2.3. For every $X \in \mathcal{A}$, let us take copies $\left\{X^{(j)}\right\}_{j \geq 1}$ in an algebraic probability space $(\widetilde{\mathcal{A}}, \mathfrak{B}, \widetilde{\varphi})$ such that

(1) $X \mapsto X^{(j)}$ is a $\mathfrak{B}$-homomorphism for each $j$;

(2) $\widetilde{\varphi}\left(X_{1}^{(j)} X_{2}^{(j)} \cdots X_{n}^{(j)}\right)=\varphi\left(X_{1} X_{2} \cdots X_{n}\right)$ for any $X_{i} \in \mathcal{A}, j, n \geq 1$; and

(3) the subalgebras $\mathcal{A}^{(j)}:=\left\{X^{(j)}\right\}_{X \in \mathcal{A}}$ are monotone independent over $\mathfrak{B}$.

We define a dot operation N.X as follows:

$$
N \cdot X=X^{(1)}+\cdots+X^{(N)}
$$

for $X \in \mathcal{A}$ and for a natural number $N \geq 0$. We understand that $0 . X=0$. The dot operation can be extended to random vectors

$$
N . X:=\left(X_{1}^{(1)}+\cdots+X_{1}^{(N)}, \ldots, X_{r}^{(1)}+\cdots+X_{r}^{(N)}\right)
$$

for $X=\left(X_{1}, \ldots, X_{r}\right)$. We can iterate the dot operation more than once in a suitable space. For instance, the symbol $N .(M . X)$ means the sum $(M . X)^{(1)}+\cdots+(M . X)^{(N)}$ of monotone i.i.d. random variables $(M . X)^{(k)}$, $k=1,2,3, \ldots$.

For simplicity, $\widetilde{\varphi}$ is denoted by the same symbol $\varphi$ in this article. The above dot operation can be realized in a canonical way in terms of a free product with amalgamation. The construction is similar to the $\mathbb{C}$-valued case in [5, Section 2], so we do not repeat it here.

An essential property of the dot operation is associativity up to a state, described by the following proposition.

Proposition 2.4. For random variables $X_{1}, \ldots, X_{n}$,

$$
\varphi\left(\left((N M) \cdot X_{1}\right) \cdots\left((N M) \cdot X_{n}\right)\right)=\varphi\left(\left(N .\left(M \cdot X_{1}\right)\right) \cdots\left(N .\left(M \cdot X_{n}\right)\right)\right) .
$$

The proof is quite similar to that of [5, Proposition 2.4].

\section{$\S 3$. Monotone cumulants}

Let us introduce terminologies and notation regarding partitions of a set. The following definitions of partitions and ordered partitions are possible on any linearly ordered set, but we consider only the set $\underline{n}:=\{1, \ldots, n\}$ for simplicity. A partition is a set $\pi=\left\{V_{1}, \ldots, V_{k}\right\}$, where $V_{i}$ are nonempty, 
disjoint subsets of $\underline{n}$ and where $\bigcup_{i=1}^{k} V_{i}=\underline{n}$. The number $k$ is denoted as $|\pi|$, and an element $V$ of $\pi$ is called a block. A partition $\pi$ is said to be crossing if blocks $V, W \in \pi$ exist so that there are elements $a, c \in V$ and $b, d \in W$ satisfying $a<b<c<d$. A partition $\pi$ is said to be noncrossing if it is not crossing. The set of the noncrossing partitions of $\underline{n}$ is denoted as $\mathcal{N C}(n)$. A block $V$ of a partition is called an interval block if $V$ is of the form $V=\{k, k+1, \ldots, k+l\}$ for $k \geq 1$ and $0 \leq l \leq n-k$. The set of the interval blocks is denoted by $I B(n)$. A partial order can be defined for partitions. For partitions $\pi$ and $\sigma$, the relation $\pi \leq \sigma$ means that for any block $V \in \pi$ there exists a block $W \in \sigma$ such that $V \subset W$. For instance, the partition consisting of one block $\{1, \ldots, n\}$ is larger than any other partition.

In addition to partitions, we need ordered partitions in this paper. An ordered partition $\pi$ of $\underline{n}$ is a sequence $\pi=\left(V_{1}, \ldots, V_{k}\right)$, where $\left\{V_{1}, \ldots, V_{k}\right\}$ is a partition of $\underline{n}$. The number $k$ is also denoted as $|\pi|$. Let us denote by $\mathcal{L} \mathcal{P}(n)$ the set of the ordered partitions of $\underline{n}$.

We introduce a partial order on blocks in a partition $\pi \in \mathcal{N C}(n)$ as follows. For $V, W \in \pi$ we denote $V \succ W$ if there are $i, j \in W$ such that $i<k<j$ for all $k \in V$. Visually, $V \succ W$ means that $V$ lies in the inner side of $W$, for instance, $\{4,5,6\} \succ\{3,8,10\}$ in Figure 1. We assume that the relation $\succ$ does not include the equality; $V \succ W$ implies that $V \neq W$ in this paper. A monotone partition of $\underline{n}$ is an ordered partition $\pi=\left(V_{1}, \ldots, V_{k}\right) \in \mathcal{L} \mathcal{P}(n)$ which satisfies the following properties:

(1) $\left\{V_{1}, \ldots, V_{k}\right\} \in \mathcal{N C}(n)$;

(2) if $V_{i} \succ V_{j}$, then $i>j$.

The set of monotone partitions of $\underline{n}$ is denoted by $\mathcal{M}(n)$.

The following definition was used by Speicher [13, Definition 2.1.1] to describe the moment-cumulant formula for the case of free probability with

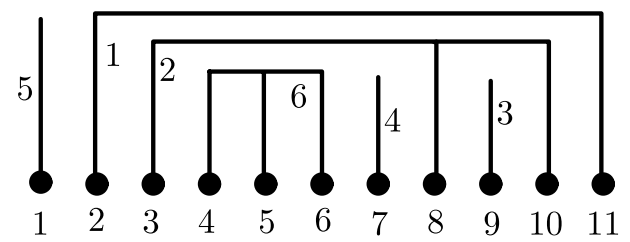

Figure 1: A monotone partition $(\{2,11\},\{3,8,10\},\{9\},\{7\},\{1\}$, $\{4,5,6\})$. Each block is labeled by a number to clarify the order on the blocks. 
amalgamation. Let $A_{n}$ be a multilinear functional from $\mathcal{A}^{n}$ to $\mathfrak{B}$ for $n \geq 1$. A multilinear functional $A_{\pi}$ for a noncrossing partition $\pi$ of $\underline{n}$ is defined by the recursive relation

$A_{\pi}\left(X_{1}, \ldots, X_{n}\right)=A_{\sigma}\left(X_{1}, \ldots, X_{k-1}, A_{m+1}\left(X_{k}, \ldots, X_{k+m}\right) X_{k+m+1}, \ldots, X_{n}\right)$,

where $V=\{k, \ldots, k+m\}$ is an interval block of $\pi$ and where $\sigma$ denotes the noncrossing partition $\pi \backslash\{V\}$ of $\underline{n} \backslash V .^{\dagger}$ If $\pi=\left(V_{1}, \ldots, V_{k}\right)$ is an ordered partition such that $\left\{V_{1}, \ldots, V_{k}\right\} \in \mathcal{N C}(n)$, then we define $A_{\pi}$ in the above way, neglecting the order structure of $\pi$.

In this article, $\varphi_{\pi}\left(X_{1}, \ldots, X_{n}\right)$ always denotes the above construction arising from the multilinear functionals $\varphi_{n}\left(X_{1}, \ldots, X_{n}\right):=\varphi\left(X_{1} \cdots X_{n}\right)$.

The following result is useful to understand an interplay among $\mathcal{L P}(n)$, $\mathcal{N C}(n)$, and monotone independence. Therefore, a detailed proof is presented.

LEMma 3.1. For each noncrossing partition $\pi$, there exists a polynomial $a_{\pi}(x)$ which does not contain a constant term such that

$$
\varphi\left(\left(N . X_{1}\right) \cdots\left(N . X_{n}\right)\right)=\sum_{\pi \in \mathcal{N C}(n)} a_{\pi}(N) \varphi_{\pi}\left(X_{1}, \ldots, X_{n}\right)
$$

for all $X_{1}, \ldots, X_{n}$ and $N \in \mathbb{N}$.

Proof. Step 1: To calculate $\varphi\left(\left(N . X_{1}\right) \cdots\left(N . X_{n}\right)\right)$, we have to know $\varphi\left(X_{1}^{\left(i_{1}\right)} \cdots X_{n}^{\left(i_{n}\right)}\right)$ for each sequence $\left(i_{1}, \ldots, i_{n}\right)$ of natural numbers. We first prove that $\varphi\left(X_{1}^{\left(i_{1}\right)} \cdots X_{n}^{\left(i_{n}\right)}\right)$ can be written as $\varphi_{\sigma}\left(X_{1}, \ldots, X_{n}\right)$ with a $\sigma \in$ $\mathcal{N C}(n)$. Let us associate an ordered partition $\pi=\left(V_{1}, \ldots, V_{p}\right)$ to a sequence $\left(i_{1}, \ldots, i_{n}\right)$ as follows. First we define $p_{1}:=\max \left\{i_{k}: 1 \leq k \leq n\right\}$ and $V_{1}:=$ $\left\{k: i_{k}=p_{1}\right\}$. Next we define $p_{2}:=\max \left\{i_{k}: k \notin V_{1}\right\}$ and $V_{2}:=\left\{k: i_{k}=p_{2}\right\}$. Recursively, we define $p_{m}:=\max \left\{i_{k}: k \notin V_{1} \cup \cdots \cup V_{m-1}\right\}$ and $V_{m}:=\{k:$ $\left.i_{k}=p_{m}\right\}$. Then we obtain an ordered partition $\pi$.

Next let us define a map $Q$ from $\mathcal{L P}(n)$ onto $\mathcal{N C}(n)$.

(1) For $\pi=\left(V_{1}, \ldots, V_{r}\right) \in \mathcal{L} \mathcal{P}(n)$, let us focus on $V_{r}$ at first. If there exists a block $V_{i}$ such that $V_{r}$ and $V_{i}$ are crossing, ${ }^{\dagger \dagger}$ then we can take the maximal partition $\sigma$ of $V_{r}$ such that no block of $\sigma$ crosses $V_{i}$. (Maximal is for the

\footnotetext{
$\dagger$ While we have not defined partitions of an arbitrary linearly ordered set, a noncrossing partition of $\underline{n} \backslash V$ can be naturally defined by using the linear order structure of $\underline{n} \backslash V$. ${ }^{\dagger \dagger}$ Blocks $V$ and $W$ are said to be crossing if there are $a, c \in V$ and $b, d \in W$ such that $a<b<c<d$ or $d<c<b<a$.
} 
partial order $\leq$.) Iterating this procedure for every $V_{i}$ crossing $V_{r}$, we finally obtain the maximal partition $\sigma_{r}$ of $V_{r}$, no block of which crosses the other blocks $V_{1}, \ldots, V_{r-1}$.

(2) We define ordered partitions $\pi_{k}:=\left(V_{1}, \ldots, V_{k}\right) \in \mathcal{L} \mathcal{P}\left(\bigcup_{i=1}^{k} V_{i}\right)$. Then we carry out the procedure (1) for $\pi_{k}$ from $k=r-1$ to $k=1$, to obtain partitions $\sigma_{k}$. Thus, we obtain a noncrossing partition $Q(\pi)$ of $\{1, \ldots, n\}$, gathering $\left\{\sigma_{k}\right\}_{k=1}^{r}$. Figures 2-4 are examples of the map $Q$. We can check that $\varphi\left(X_{1}^{\left(i_{1}\right)} \cdots X_{n}^{\left(i_{n}\right)}\right)$ is equal to $\varphi_{Q(\pi)}\left(X_{1}, \ldots, X_{n}\right)$ if $\pi$ denotes the ordered partition associated to $\left(i_{1}, \ldots, i_{n}\right)$.

Step 2: The remaining proof is similar to the $\mathbb{C}$-valued case, based on induction. For $n=1, \varphi\left(N . X_{1}\right)=N \varphi\left(X_{1}\right)$, so that the assertion is true. We

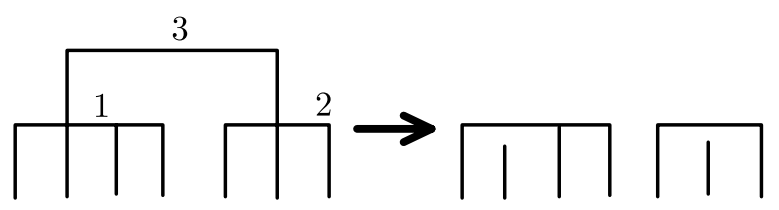

Figure 2: The ordered partition $(\{1,3,4\},\{5,7\},\{2,6\})$ is mapped to the noncrossing partition $\{\{1\},\{2,6\},\{3,4\},\{5\},\{7\}\}$.

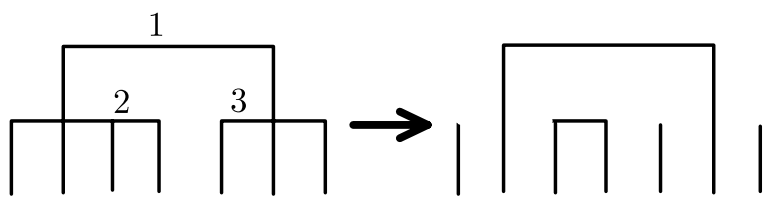

Figure 3: The ordered partition $(\{2,6\},\{1,3,4\},\{5,7\})$ is mapped to the noncrossing partition $\{\{1\},\{2,6\},\{3,4\},\{5\},\{7\}\}$.

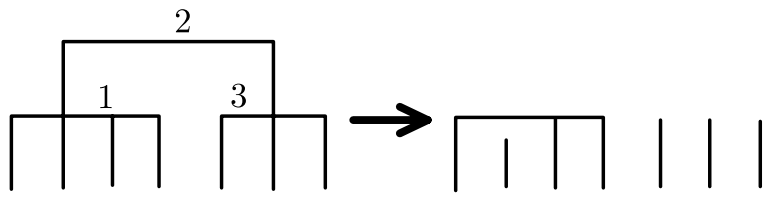

Figure 4: The ordered partition $(\{1,3,4\},\{2,6\},\{5,7\})$ is mapped to the noncrossing partition $\{\{1,3,4\},\{2\},\{5\},\{6\},\{7\}\}$. 
assume that the assertion is true for $n$. The identity

$$
\begin{gathered}
\varphi\left(\left(X_{1}+Y_{1}\right) \cdots\left(X_{n+1}+Y_{n+1}\right)\right)-\varphi\left(Y_{1} \cdots Y_{n+1}\right) \\
=\sum_{\substack{Z_{i} \in\left\{X_{i}, Y_{i}\right\}, 1 \leq i \leq n+1, Z_{j}=Y_{j} \text { for some } j}} \varphi\left(Z_{1} \cdots Z_{n+1}\right)
\end{gathered}
$$

holds for any random variables $X_{i}, Y_{i}$. We assume that random vectors $\left(X_{1}, \ldots, X_{n+1}\right)$ and $\left(Y_{1}, \ldots, Y_{n+1}\right)$ are monotone independent over $\mathfrak{B}$. Then each term $\varphi\left(Z_{1} \cdots Z_{n+1}\right)$ factorizes by using monotone independence, and expectations $\varphi\left(Y_{k} Y_{k+1} \cdots Y_{k+m-1}\right)$ appear satisfying $1 \leq m \leq n$. Let us replace $X_{i}$ by $X_{i}^{(1)}$ and $Y_{i}$ by $X_{i}^{(2)}+\cdots+X_{i}^{(M)}$ in $\varphi\left(\left(X_{1}+Y_{1}\right) \cdots\left(X_{n+1}+\right.\right.$ $\left.\left.Y_{n+1}\right)\right)$. Then the difference $\varphi\left(M . X_{1} \cdots M . X_{n+1}\right)-\varphi\left((M-1) \cdot X_{1} \cdots\right.$ $\left.(M-1) \cdot X_{n+1}\right)$ can be written as

$$
\sum_{\pi \in \mathcal{N C}(n+1)} b_{\pi}(M) \varphi_{\pi}\left(X_{1}, \ldots, X_{n+1}\right)
$$

for some polynomials $b_{\pi}(x)$, by using the assumption of induction. The above sum is taken over only noncrossing partitions because of the assumption of induction and Step 1. By summing up (3.1) over $M$ from 1 to $N$, we conclude the assertion for $n+1$ since $\sum_{M=1}^{N} M^{p}$ is a polynomial on $N$ of degree $p+1$ without a constant term.

REMARK 3.2. The coefficients $a_{\pi}(N)$ are universal in the sense that they do not depend on a choice of noncommutative probability spaces $(\mathcal{A}, \mathfrak{B}, \varphi)$ and $(\widetilde{\mathcal{A}}, \mathfrak{B}, \widetilde{\varphi})$ of Definition 2.3.

Definition 3.3. We define an $n$th joint cumulant $K_{n}\left(X_{1}, \ldots, X_{n}\right)$ as the coefficient of $N$ appearing in $\varphi\left(\left(N . X_{1}\right)\left(N . X_{2}\right) \cdots\left(N . X_{n}\right)\right)$. Note that $K_{n}\left(X_{1}, \ldots, X_{n}\right)$ is also written as $K_{n}^{X}$ with $X=\left(X_{1}, \ldots, X_{n}\right)$ for simplicity. The arguments in [5, Proposition 5.1, Corollary 5.2, Theorem 5.3] can be easily extended to the operator-valued setting, and then we obtain the following.

THEOREM 3.4. The following moment-cumulant formula holds:

$$
\varphi\left(X_{1} \cdots X_{n}\right)=\sum_{\pi \in \mathcal{M}(n)} \frac{1}{|\pi| !} K_{\pi}\left(X_{1}, \ldots, X_{n}\right) .
$$

From Proposition 2.4, one can prove the following additivity property of cumulants for monotone i.i.d. random variables. 
Proposition 3.5. For $X=\left(X_{1}, \ldots, X_{n}\right) \in \mathcal{A}^{n}$,

$$
K_{n}^{N \cdot X}=N K_{n}^{X} \text {. }
$$

The central limit theorem was considered in [10, Theorem 5.3], and its limit distribution was further studied in [1]. As an application of cumulants, we can obtain an easy proof and a different formula of the limit distribution using an argument quite similar to that in [6, Theorem 5.1].

THEOREM 3.6. Let $\left(X_{i}\right)_{i=1}^{\infty}$ be monotone i.i.d. random variables. We assume that $\varphi\left(X_{i}\right)=0$ for any $i$, and we define $X(N):=\left(X_{1}+\cdots+X_{N}\right) /$ $\sqrt{N}$. Then

$$
\begin{aligned}
\lim _{N \rightarrow \infty} \varphi\left(b_{1} X(N) b_{2} \cdots b_{n} X(N)\right) \\
\quad= \begin{cases}\sum_{\pi \in \mathcal{M}_{2}(n) \frac{1}{|\pi| !} K_{\pi}\left(b_{1} X_{1}, \ldots, b_{n} X_{1}\right)} & \text { if } n \text { is even, } \\
0 & \text { if } n \text { is odd, }\end{cases}
\end{aligned}
$$

where $\mathcal{M}_{2}(2 k)$ is the set of monotone pair partitions $\{\pi \in \mathcal{M}(2 k):|V|=$ 2 for any block $V$ of $\pi$ \}.

\section{§. Generating functions}

One purpose of this article is to investigate generating functions of moments and monotone cumulants of multivariate random variables $X=\left(X_{1}, \ldots, X_{r}\right)$. For $\mathfrak{B}=\mathbb{C}$ and $r=1$, let us consider $M^{X}(z)=1+$ $\sum_{n=1}^{\infty} \varphi\left(X^{n}\right) z^{n}$ and $\mu^{X}(z):=z M^{X}(z)$. In [9, Theorem 3.1] Muraki proved that $\mu^{X+Y}(z)=\mu^{X}\left(\mu^{Y}(z)\right)$ for monotone independent random variables $X$ and $Y$. This relation can also be written as

$$
M^{X+Y}(z)=M^{X}\left(z M^{Y}(z)\right) M^{Y}(z) .
$$

In [10, Theorem 3.7], Popa proved an analogue of this formula for general $\mathfrak{B}$ and $r=1$. Muraki's formula was also extended to general $r$ with $\mathfrak{B}=\mathbb{C}$ (see [5, Corollary 6.2]). In the following, we are going to prove the most general version, that is, for general $\mathfrak{B}$ and $r$.

This section is also related to the work of Dykema [3], in which the ring $\mathbb{C} \llbracket z \rrbracket$ of formal power series was extended to the operator-valued case. A key in this extension is to replace a formal power series by a sequence of multilinear functionals. Dykema introduced a composition operation and investigated an algebraic structure of such multilinear functionals.

In this section, we generalize such an algebraic structure to the multivariate case. In other words, we generalize the ring $\mathbb{C} \llbracket z_{1}, \ldots, z_{r} \rrbracket$ generated 
by free indeterminates to the operator-valued case. Let us consider how to extend the composition of two functions. We generalize not the usual composition operation $\circ$ but a modified associative operation

$$
(F \bullet G)(z):=F(z G(z)) G(z) .
$$

This is natural for monotone independence, as we can guess from (4.1).

First we extend the ring $\mathbb{C} \llbracket z_{1}, \ldots, z_{r} \rrbracket$ to include the $\mathfrak{B}$-valued case.

Definition 4.1. In this given case, we define the set $\mathrm{Mul}^{r} \llbracket \mathfrak{B} \rrbracket$ of all $F=$ $\left(F_{i_{1}, \ldots, i_{n}}\right)_{i_{1}, \ldots, i_{n} \in \underline{r}, n \geq 0}$, where $F_{i_{1}, \ldots, i_{n}}$ is a multilinear functional from $\mathfrak{B}^{n}$ to $\mathfrak{B}$, and where $n=0$ corresponds to a constant $F_{\emptyset} \in \mathfrak{B}$.

To define an analogue of the composition, we need some notation and concepts.

Definition 4.2. Let $V$ be a subset of $\underline{n}$, denoted as $\left\{v_{1}, v_{2}, \ldots, v_{p}\right\}$, where $1 \leq v_{1}<\cdots<v_{p} \leq n$. We moreover add edges $v_{0}:=0$ and $v_{p+1}:=n+1$ for convenience.

(1) The interval blocks $V_{i}=\left\{v_{i-1}+1, \ldots, v_{i}-1\right\}$ for $1 \leq i \leq p+1$ are called the interpolation blocks of $V$ for $\underline{n}$. If $v_{i-1}+1=v_{i}$, we set $V_{i}:=\emptyset$. If $V=\emptyset$, we consider only one interpolation block $V_{1}=\underline{n}$. Clearly, this notion can be extended for the case of any linearly ordered set.

(2) Let $V=\left\{v_{1}, \ldots, v_{p}\right\}$ be a subset of $\underline{n}$ with $v_{1}<\cdots<v_{p}$. For a tuple $\left(i_{1}, \ldots, i_{n}\right) \in \mathbb{N}^{n}$, we define $i_{V}=i(V):=\left(i_{v_{1}}, \ldots, i_{v_{p}}\right)$. If $V=\emptyset$, then $i(V):=\emptyset$. For a multilinear functional $F_{p}: \mathfrak{B}^{p} \rightarrow \mathfrak{B}$, we set $F_{p}\left(b_{V}\right):=$ $F_{p}\left(b_{v_{1}}, \ldots, b_{v_{p}}\right)$.

\section{EXAMPLE 4.3.}

(1) If $V=\{2,3,4,6\} \subset \underline{6}$, the interpolation blocks of $V$ for $\underline{6}$ are given by $V_{1}=\{1\}, V_{2}=V_{3}=V_{5}=\emptyset, V_{4}=\{5\}$.

(2) Let $\{1,2,3,4,6,7,8\}$ be endowed with the natural order structure. If $V=$ $\{3,4,7\}$, then the interpolation blocks of $V$ for $\{1,2,3,4,6,7,8\}$ are given by $V_{1}=\{1,2\}, V_{2}=\emptyset, V_{3}=\{6\}, V_{4}=\{8\}$.

Now we introduce algebraic structure on $\mathrm{Mul}^{r} \llbracket \mathfrak{B} \rrbracket$ and some operations. 
DEFINITION 4.4.

(1) For $F, G \in \mathrm{Mul}^{r} \llbracket \mathfrak{B} \rrbracket, F \odot G \in \mathrm{Mul}^{r} \llbracket \mathfrak{B} \rrbracket$ is defined by

$$
\left\{\begin{aligned}
(F \odot & \odot)_{i_{1}, \ldots, i_{n}}\left(b_{1}, \ldots, b_{n}\right) \\
& :=\sum_{V=\left\{v_{1}, v_{2}, \ldots, v_{p}\right\} \subset \underline{n}} F_{i(V)}\left(G_{i\left(V_{1}\right)}\left(b_{V_{1}}\right) b_{v_{1}}, \ldots, G_{i\left(V_{p}\right)}\left(b_{V_{p}}\right) b_{v_{p}}\right) \\
& \times G_{i\left(V_{p+1}\right)}\left(b_{V_{p+1}}\right), \quad n \geq 1, \\
(F \odot & G)_{\emptyset}:=F_{\emptyset} G_{\emptyset},
\end{aligned}\right.
$$

where $V_{i}$ are the interpolation blocks of $V$ for $\underline{n}$. If an interpolation block $V_{j}$ is empty, then $G_{i\left(V_{j}\right)}\left(b_{V_{j}}\right)$ is understood to be $G_{\emptyset}$. The sum over $V \subset \underline{n}$ includes the case $V=\emptyset$; in this case $F_{i(V)}\left(G_{i\left(V_{1}\right)}\left(b_{V_{1}}\right) b_{v_{1}}, \ldots\right.$, $\left.G_{i\left(V_{p}\right)}\left(b_{V_{p}}\right) b_{v_{p}}\right) G_{i\left(V_{p+1}\right)}\left(b_{V_{p+1}}\right)$ is understood to be $F_{\emptyset} G_{i_{1}, \ldots, i_{n}}\left(b_{1}, \ldots, b_{n}\right)$.

(2) Let $F(t) \in \mathrm{Mul}^{r} \llbracket \mathfrak{B} \rrbracket$ for $t \in \mathbb{R}$ such that each $F(t)_{i_{1}, \ldots, i_{n}}$ is differentiable with respect to $t$. Then we define $\frac{d F}{d t}(t) \in \mathrm{Mul}^{r} \llbracket \mathfrak{B} \rrbracket$ to be $\left(\frac{d}{d t} F(t)_{i_{1}, \ldots, i_{n}}\right)_{i_{1}, \ldots, i_{n} \in \underline{r}, n \geq 0}$.

(3) We define a binary operation $\star$ on $\mathrm{Mul}^{r} \llbracket \mathfrak{B} \rrbracket$ by

$$
\begin{aligned}
(F \star G)_{i_{1}, \ldots, i_{n}}\left(b_{1}, \ldots, b_{n}\right) & :=\sum_{V=\{k, \ldots, k+l\} \in I B(n)} F_{i\left(V^{c}\right)}\left(b_{1}, \ldots, b_{k-1}, G_{i(V)}\left(b_{V}\right) b_{k+l+1}, \ldots, b_{n}\right) .
\end{aligned}
$$

If $k+l=n$, the summand is understood to be $F_{i\left(V^{c}\right)}\left(b_{V^{c}}\right) G_{i(V)}\left(b_{V}\right)$. For $n=0$, we define $(F \star G)_{\emptyset}:=F_{\emptyset} G_{\emptyset}$.

REMARK 4.5 .

(1) The operation $\odot$ corresponds to the modified composition (4.2). Moreover, a relation to the paper [5] is as follows. To treat generating functions related to random vectors with $\mathfrak{B}=\mathbb{C}$, we use the formal power series

$$
A\left(z_{1}, \ldots, z_{r}\right)=a_{\emptyset}+\sum_{n=1}^{\infty} \sum_{i_{1}, \ldots, i_{n}=1}^{r} a_{i_{1}, \ldots, i_{n}} z_{i_{1}} \cdots z_{i_{n}},
$$

where $a_{\emptyset}, a_{i_{1}, \ldots, i_{n}} \in \mathbb{C}$ and $z_{1}, \ldots, z_{r}$ are free generators. Let us denote by $\mathbb{C} \llbracket z_{1}, \ldots, z_{r} \rrbracket$ the set of such formal power series. Then we can define an associative product $\bullet$ as $F \bullet G:=S^{-1}((S F) \circ(S G))$, where $S F\left(z_{1}, \ldots\right.$, $\left.z_{r}\right):=\left(z_{1} F\left(z_{1}, \ldots, z_{r}\right), \ldots, z_{r} F\left(z_{1}, \ldots, z_{r}\right)\right)$. This operation was essentially defined in $[5$, Corollary 6.2$]$. For a given $A \in \mathbb{C} \llbracket z_{1}, \ldots, z_{r} \rrbracket$ of the form (4.3), we can associate $\widetilde{A} \in \mathrm{Mul}^{r} \llbracket \mathbb{C} \rrbracket$ by defining $\widetilde{A}_{i_{1}, \ldots, i_{n}}\left(b_{1}, \ldots\right.$, $\left.b_{n}\right):=a_{i_{1}, \ldots, i_{n}} b_{1} \cdots b_{n}\left(b_{i} \in \mathbb{C}\right)$. Then the operation $\odot$ coincides with $\bullet$. 
(2) The operation $\star$ appears when we take a derivative of $F \circ H(t)$ regarding $\left.\frac{d}{d t}\right|_{0}$ under the condition $H(0)=0$.

EXAMPLE 4.6.

(1) For $n=1$, we have $(F \odot G)_{i_{1}}\left(b_{1}\right)=F_{i_{1}}\left(G_{\emptyset} b_{1}\right) G_{\emptyset}+F_{\emptyset} G_{i_{1}}\left(b_{1}\right)$.

(2) For $n=2$, we have $(F \odot G)_{i_{1}, i_{2}}\left(b_{1}, b_{2}\right)=F_{i_{1}, i_{2}}\left(G_{\emptyset} b_{1}, G_{\emptyset} b_{2}\right) G_{\emptyset}+$ $F_{i_{1}}\left(G_{\emptyset} b_{1}\right) G_{i_{2}}\left(b_{2}\right)+F_{i_{2}}\left(G_{i_{1}}\left(b_{1}\right) b_{2}\right) G_{\emptyset}+F_{\emptyset} G_{i_{1}, i_{2}}\left(b_{1}, b_{2}\right)$.

(3) For $n=3$, we have $(F \odot G)_{i_{1}, i_{2}, i_{3}}\left(b_{1}, b_{2}, b_{3}\right)=F_{i_{1}, i_{2}, i_{3}}\left(G_{\emptyset} b_{1}, G_{\emptyset} b_{2}\right.$, $\left.G_{\emptyset} b_{3}\right) G_{\emptyset}+F_{i_{1}, i_{2}}\left(G_{\emptyset} b_{1}, G_{\emptyset} b_{2}\right) G_{i_{3}}\left(b_{3}\right)+F_{i_{1}, i_{3}}\left(G_{\emptyset} b_{1}, G_{i_{2}}\left(b_{2}\right) b_{3}\right) G_{\emptyset}+$ $F_{i_{2}, i_{3}}\left(G_{i_{1}}\left(b_{1}\right) b_{2}, G_{\emptyset} b_{3}\right) G_{\emptyset}+F_{i_{1}}\left(G_{\emptyset} b_{1}\right) G_{i_{2}, i_{3}}\left(b_{2}, b_{3}\right)+F_{i_{2}}\left(G_{i_{1}}\left(b_{1}\right) b_{2}\right) \times$ $G_{i_{3}}\left(b_{3}\right)+F_{i_{3}}\left(G_{i_{1}, i_{2}}\left(b_{1}, b_{2}\right) b_{3}\right) G_{\emptyset}+F_{\emptyset} G_{i_{1}, i_{2}, i_{3}}\left(b_{1}, b_{2}, b_{3}\right)$.

In general, $(F \odot G)_{i_{1}, \ldots, i_{n}}\left(b_{1}, \ldots, b_{n}\right)$ can be written as the sum of $2^{n}$ terms.

\section{Proposition 4.7.}

(1) The composition $\odot$ is associative: $(F \odot G) \odot H=F \odot(G \odot H)$ for any $F, G, H \in \mathrm{Mul}^{r} \llbracket \mathfrak{B} \rrbracket$.

(2) The element $\mathrm{Id}:=\left(\operatorname{Id}_{i_{1}, \ldots, i_{n}}\right)$, defined by $\operatorname{Id}_{\emptyset}=1$ and $\operatorname{Id}_{i_{1}, \ldots, i_{n}}=0$ for $n \geq 1$, is the identity for the operation $\odot: \operatorname{Id} \odot F=F \odot \operatorname{Id}=F$ for any $F \in \mathrm{Mul}^{r} \llbracket \mathfrak{B} \rrbracket$.

(3) The left distributive law $(F+G) \odot H=F \odot H+G \odot H$ holds for any $F, G, H \in \mathrm{Mul}^{r} \llbracket \mathfrak{B} \rrbracket$.

Proof. (1) We fix a tuple $\left(i_{1}, \ldots, i_{n}\right)$, and we will prove the following:

$$
((F \odot G) \odot H)_{i_{1}, \ldots, i_{n}}\left(b_{1}, \ldots, b_{n}\right)=(F \odot(G \odot H))_{i_{1}, \ldots, i_{n}}\left(b_{1}, \ldots, b_{n}\right) .
$$

For each sequence of words $\{F, G, H\}$ of length $n$, one can associate an $n$-linear functional as follows. Given a sequence $\left(A_{1}, \ldots, A_{n}\right)$, where each $A_{j}$ is equal to $F, G$, or $H$, we gather the indices $j$ such that $A_{j}=F$ and denote them by $j_{1}<j_{2}<\cdots<j_{p}$. Let $J_{s}$ be the interpolation blocks $(s=1, \ldots, p+1)$ of $J:=\left\{j_{1}, \ldots, j_{p}\right\}$ for $\underline{n}$. If $J$ is the empty set, then we understand that $p=0$ and $J_{1}=\underline{n}$. For each $s$, let us denote all the indices $k \in J_{s}$ such that $A_{k}=G$ by $k_{1}^{(s)}<\cdots<k_{q(s)}^{(s)}$. Let $K_{m}^{(s)}(m=1, \ldots, q(s)+1)$ be the interpolation blocks of $K^{(s)}:=\left\{k_{1}^{(s)}, \ldots, k_{q(s)}^{(s)}\right\}$ for the linearly ordered set $J_{s}$. Now we define 


$$
I_{s}\left(b_{J_{s}}\right):= \begin{cases}G_{i\left(K^{(s)}\right)}\left(H_{i\left(K_{1}^{(s)}\right)}\left(b_{K_{1}^{(s)}}\right) b_{k_{1}^{(s)}}, H_{i\left(K_{2}^{(s)}\right)}\left(b_{K_{2}^{(s)}}\right) b_{k_{2}^{(s)}},\right. & \\ \ldots, H_{i\left(K_{q(s)}^{(s)}\right)}\left(b_{\left.K_{q(s)}^{(s)}\right)} b_{\left.k_{q(s)}^{(s)}\right)} H_{i\left(K_{q(s)+1}^{(s)}\right)}\left(b_{\left.K_{q(s)+1}^{(s)}\right)}\right)\right. & \text { if } K^{(s)} \neq \emptyset, \\ G_{\emptyset} H_{i\left(K_{q(s)+1}^{(s)}\right)}\left(b_{\left.K_{q(s)+1}^{(s)}\right),}\right. & \text { if } K^{(s)}=\emptyset .\end{cases}
$$

We understand that $H_{i\left(K_{m}^{(s)}\right)}\left(b_{K_{m}^{(s)}}\right)=H_{\emptyset} \in \mathfrak{B}$ if $K_{m}^{(s)}=\emptyset$. Using $I_{s}$, we define an $n$-linear functional

$$
\begin{aligned}
L_{\left(A_{1}, \ldots, A_{n}\right)}\left(b_{1}, \ldots, b_{n}\right) \\
:= \begin{cases}F_{i_{1}, \ldots, i_{n}}\left(I_{1}\left(b_{J_{1}}\right) b_{j_{1}}, \ldots, I_{p}\left(b_{J_{p}}\right) b_{j_{p}}\right) I_{p+1}\left(b_{J_{p+1}}\right) & \text { if } J \neq \emptyset, \\
F_{\emptyset} I_{p+1}\left(b_{J_{p+1}}\right), & \text { if } J=\emptyset .\end{cases}
\end{aligned}
$$

Examples of $L_{\left(A_{1}, \ldots, A_{n}\right)}$ can be found in Tables 1-4 below.

Table 1: The elements of $\mathcal{L}_{1}$.

\begin{tabular}{|l|l|}
\hline$\left(A_{1}\right)$ & $L_{\left(A_{1}\right)}\left(b_{1}\right)$ \\
\hline$(F)$ & $F_{i_{1}}\left(G_{\emptyset} H_{\emptyset} b_{1}\right) G_{\emptyset} H_{\emptyset}$ \\
\hline$(G)$ & $F_{\emptyset} G_{i_{1}}\left(H_{\emptyset} b_{1}\right) H_{\emptyset}$ \\
\hline$(H)$ & $F_{\emptyset} G_{\emptyset} H_{i_{1}}\left(b_{1}\right)$ \\
\hline
\end{tabular}

Table 2: The elements of $\mathcal{L}_{2}$.

\begin{tabular}{|l|l|}
\hline$\left(A_{1}, A_{2}\right)$ & $L_{\left(A_{1}, A_{2}\right)}\left(b_{1}, b_{2}\right)$ \\
\hline$(F, F)$ & $F_{i_{1}, i_{2}}\left(G_{\emptyset} H_{\emptyset} b_{1}, G_{\emptyset} H_{\emptyset} b_{2}\right) G_{\emptyset} H_{\emptyset}$ \\
\hline$(F, G)$ & $F_{i_{1}}\left(G_{\emptyset} H_{\emptyset} b_{1}\right) G_{i_{2}}\left(H_{\emptyset} b_{2}\right) H_{\emptyset}$ \\
\hline$(F, H)$ & $F_{i_{1}}\left(G_{\emptyset} H_{\emptyset} b_{1}\right) G_{\emptyset} H_{i_{2}}\left(b_{2}\right)$ \\
\hline$(G, F)$ & $F_{i_{2}}\left(G_{i_{1}}\left(H_{\emptyset} b_{1}\right) H_{\emptyset} b_{2}\right) G_{\emptyset} H_{\emptyset}$ \\
\hline$(G, G)$ & $F_{\emptyset} G_{i_{1}, i_{2}}\left(H_{\emptyset} b_{1}, H_{\emptyset} b_{2}\right) H_{\emptyset}$ \\
\hline$(G, H)$ & $F_{\emptyset} G_{i_{1}}\left(H_{\emptyset} b_{1}\right) H_{i_{2}}\left(b_{2}\right)$ \\
\hline$(H, F)$ & $F_{i_{2}}\left(G_{\emptyset} H_{i_{1}}\left(b_{1}\right) b_{2}\right) G_{\emptyset} H_{\emptyset}$ \\
\hline$(H, G)$ & $F_{\emptyset} G_{i_{2}}\left(H_{i_{1}}\left(b_{1}\right) b_{2}\right) H_{\emptyset}$ \\
\hline$(H, H)$ & $F_{\emptyset} G_{\emptyset} H_{i_{1}, i_{2}}\left(b_{1}, b_{2}\right)$ \\
\hline
\end{tabular}


Table 3: Selected elements of $\mathcal{L}_{n}$ for $n=3,4$.

\begin{tabular}{|l|l|}
\hline Word sequence & Multilinear functional \\
\hline$(F, F, G)$ & $F_{i_{1}, i_{2}}\left(G_{\emptyset} H_{\emptyset} b_{1}, G_{\emptyset} H_{\emptyset} b_{2}\right) G_{i_{3}}\left(H_{\emptyset} b_{3}\right) H_{\emptyset}$ \\
\hline$(F, G, F)$ & $F_{i_{1}, i_{3}}\left(G_{\emptyset} H_{\emptyset} b_{1}, G_{i_{2}}\left(H_{\emptyset} b_{2}\right) H_{\emptyset} b_{3}\right) G_{\emptyset} H_{\emptyset}$ \\
\hline$(F, H, G, F)$ & $F_{i_{1}, i_{4}}\left(G_{\emptyset} H_{\emptyset} b_{1}, G_{i_{3}}\left(H_{i_{2}}\left(b_{2}\right) b_{3}\right) H_{\emptyset} b_{4}\right) G_{\emptyset} H_{\emptyset}$ \\
\hline$(F, G, F, H)$ & $F_{i_{1}, i_{3}}\left(G_{\emptyset} H_{\emptyset} b_{1}, G_{i_{2}}\left(H_{\emptyset} b_{2}\right) H_{\emptyset} b_{3}\right) G_{\emptyset} H_{i_{4}}\left(b_{4}\right)$ \\
\hline$(H, G, H, G)$ & $F_{\emptyset} G_{i_{2}, i_{4}}\left(H_{i_{1}}\left(b_{1}\right) b_{2}, H_{i_{3}}\left(b_{3}\right) b_{4}\right) H_{\emptyset}$ \\
\hline$(H, G, F, G)$ & $F_{i_{3}}\left(G_{i_{2}}\left(H_{i_{1}}\left(b_{1}\right) b_{2}\right) H_{\emptyset} b_{3}\right) G_{i_{4}}\left(H_{\emptyset} b_{4}\right) H_{\emptyset}$ \\
\hline
\end{tabular}

Table 4: Selected elements of $\mathcal{L}_{n}$ for $n \geq 5$.

\begin{tabular}{|l|l|}
\hline Word sequence & Multilinear functional \\
\hline$(F, H, G, F, F)$ & $F_{i_{1}, i_{4}, i_{5}}\left(G_{\emptyset} H_{\emptyset} b_{1}, G_{i_{3}}\left(H_{i_{2}}\left(b_{2}\right) b_{3}\right) H_{\emptyset} b_{4}, G_{\emptyset} H_{\emptyset} b_{5}\right) G_{\emptyset} H_{\emptyset}$ \\
\hline$(G, G, F, H, F, F)$ & $F_{i_{3}, i_{5}, i_{6}}\left(G_{i_{1}, i_{2}}\left(H_{\emptyset} b_{1}, H_{\emptyset} b_{2}\right) H_{\emptyset} b_{3}, G_{\emptyset} H_{i_{4}}\left(b_{4}\right) b_{5}, G_{\emptyset} H_{\emptyset} b_{6}\right) G_{\emptyset} H_{\emptyset}$ \\
\hline$(G, H, H, F, G, F, H, H)$ & $F_{i_{4}, i_{6}}\left(G_{i_{1}}\left(H_{\emptyset} b_{1}\right) H_{i_{2}, i_{3}}\left(b_{2}, b_{3}\right) b_{4}, G_{i_{5}}\left(H_{\emptyset} b_{5}\right) H_{\emptyset} b_{6}\right) G_{\emptyset} H_{i_{7}, i_{8}}\left(b_{7}, b_{8}\right)$ \\
\hline
\end{tabular}

Let us denote by $\mathcal{L}_{n}$ the set $\left\{L_{\left(A_{1}, \ldots, A_{n}\right)} \mid A_{i} \in\{F, G, H\}, 1 \leq i \leq n\right\}$, regarding the multilinear functionals $F_{i_{1}, \ldots, i_{k}}, G_{i_{1}, \ldots, i_{k}}, H_{i_{1}, \ldots, i_{k}}$ as indeterminates. Then the map $\left(A_{1}, \ldots, A_{n}\right) \mapsto L_{\left(A_{1}, \ldots, A_{n}\right)}$ is one-to-one to a set of indeterminates, and hence the cardinality of $\mathcal{L}_{n}$ is $3^{n}$. The multilinear functional $((F \odot G) \odot H)_{i_{1}, \ldots, i_{n}}\left(b_{1}, \ldots, b_{n}\right)$ can be expanded as the sum of distinct elements of $\mathcal{L}_{n}$ by definition, and moreover, the number of those elements is $\sum_{i}\left(\begin{array}{c}n-i \\ i\end{array}\right) 2^{i}=3^{n}$. So every element of $\mathcal{L}_{n}$ appears just once in $((F \odot G) \odot H)_{i_{1}, \ldots, i_{n}}\left(b_{1}, \ldots, b_{n}\right)$, which means that $((F \odot G) \odot H)_{i_{1}, \ldots, i_{n}}=$ $\sum_{L \in \mathcal{L}_{n}} L$. A similar reasoning implies that $(F \odot(G \odot H))_{i_{1}, \ldots, i_{n}}=\sum_{L \in \mathcal{L}_{n}} L$. So we can conclude that $((F \odot G) \odot H)_{i_{1}, \ldots, i_{n}}=(F \odot(G \odot H))_{i_{1}, \ldots, i_{n}}$.

Assertions (2) and (3) are not difficult.

\section{Definition 4.8.}

(1) We define a generating function of joint moments by

$$
\mu^{X}:=\left(\mu_{i_{1}, \ldots, i_{n}}^{X}\right)_{i_{1}, \ldots, i_{n} \in \underline{r}, n \geq 0} \in \mathrm{Mul}^{r} \llbracket \mathfrak{B} \rrbracket
$$

For $n=0, \mu_{\emptyset}^{X}$ is defined to be the unit $1_{\mathfrak{B}}$. 
(2) A generating function of cumulants $\kappa^{X} \in \mathrm{Mul}^{r} \llbracket \mathfrak{B} \rrbracket$ for $X=\left(X_{1}, \ldots, X_{r}\right)$ is defined by

$$
\left\{\begin{array}{l}
\kappa_{i_{1}, \ldots, i_{n}}^{X}\left(b_{1}, \ldots, b_{n}\right):=K_{n}\left(b_{1} X_{i_{1}}, \ldots, b_{n} X_{i_{n}}\right), \quad n \geq 1, \\
\kappa_{\emptyset}^{X}:=0 .
\end{array}\right.
$$

The following theorem extends formulas of [10, Theorem 3.7] and [5, Corollary 6.2] to the sum of independent random vectors.

Theorem 4.9. (Extended Muraki's formula) Let $X=\left(X_{1}, \ldots, X_{r}\right)$ and $Y=\left(Y_{1}, \ldots, Y_{r}\right)$ be random vectors which are monotone independent over $\mathfrak{B}$. Then

$$
\mu^{X+Y}=\mu^{X} \odot \mu^{Y}
$$

Proof. For simplicity, we assume that $\left(i_{1}, \ldots, i_{n}\right)=(1, \ldots, n)$ and prove that $\mu_{1, \ldots, n}^{X+Y}=\left(\mu^{X} \odot \mu^{Y}\right)_{1, \ldots, n}$. The left-hand side is

$$
\begin{aligned}
\mu_{1, \ldots, n}^{X+Y}\left(b_{1}, \ldots, b_{n}\right) & =\varphi\left(b_{1}\left(X_{1}+Y_{1}\right) b_{2} \cdots b_{n}\left(X_{n}+Y_{n}\right)\right) \\
& =\sum_{Z_{i} \in\left\{X_{i}, Y_{i}\right\}, 1 \leq i \leq n} \varphi\left(b_{1} Z_{1} b_{2} \cdots b_{n} Z_{n}\right)
\end{aligned}
$$

For each summand, let us denote by $V$ the positions where $X_{i}$ are taken, that is, $V=\left\{i: Z_{i}=X_{i}\right\}$, and write $V=\left\{v_{1}, \ldots, v_{p}\right\}, v_{1}<\cdots<v_{p}$. By using the interpolation blocks of $V$ for $\underline{n}$, the summand can be written as

$$
\mu_{V}^{X}\left(\mu_{V_{1}}^{Y}\left(b_{V_{1}}\right) b_{v_{1}}, \ldots, \mu_{V_{p}}^{Y}\left(b_{V_{p}}\right) b_{v_{p}}\right) \mu_{V_{p+1}}^{Y}\left(b_{V_{p+1}}\right) .
$$

Therefore, $\mu_{1, \ldots, n}^{X+Y}\left(b_{1}, \ldots, b_{n}\right)$ is equal to the summation of (4.4) over $V \subset \underline{n}$. By definition, this is equal to $\left(\mu^{X} \odot \mu^{Y}\right)_{1, \ldots, n}$.

Using Lemma 3.1, one can define $\mu^{t . X}$ by extending $N$ of $\mu^{N . X}$ to $t \in \mathbb{R}$. Then we obtain differential equations.

Corollary 4.10. Let $X=\left(X_{1}, \ldots, X_{r}\right)$ be a random vector. Then

$$
\frac{d}{d t} \mu^{t . X}=\kappa^{X} \odot \mu^{t . X}=\mu^{t . X} \star \kappa^{X} .
$$

Proof. The equality $\mu^{(M+N) \cdot X}=\mu^{N \cdot X} \odot \mu^{M . X}$ follows from Theorem 4.9, with $X$ replaced by $X^{(1)}+\cdots+X^{(N)}$ and $Y$ by $X^{(N+1)}+\cdots+X^{(N+M)}$. Lemma 3.1 implies that $\mu_{i_{1}, \ldots, i_{n}}^{N . X}$ is a polynomial of $N$, so that this is an identity as polynomials with respect to $N$ and $M$. Therefore, $N$ and $M$ can be replaced by real numbers $t$ and $s$, respectively. The derivatives $\left.\frac{d}{d t}\right|_{0}$ and $\left.\frac{d}{d s}\right|_{0}$ then yield the first and second identities, respectively. 
The above two differential equations can be used to calculate monotone cumulants from moments. If $\mathfrak{B}=\mathbb{C}$ and $r=1$, the above relations just coincide with

$$
\frac{d}{d t} F_{t}^{X}(z)=A^{X}\left(F_{t}^{X}(z)\right)=A^{X}(z) \frac{\partial F_{t}^{X}}{\partial z}(z)
$$

where $F_{t}^{X}(z)$ is the reciprocal Cauchy transform of a formal convolution semigroup associated to $X$ and where $A^{X}(z)=-\sum_{n=1}^{\infty}\left(K_{n}(X)\right) /\left(z^{n-1}\right)$ is a generating function of monotone cumulants. The reader is referred to the last remark of [5] for details.

Acknowledgments. Takahiro Hasebe thanks Mihai Popa for many discussions which improved his understanding of operator-valued independence. Hasebe's work was partially supported by the Global Centers of Excellence program at Kyoto University.

\section{REFERENCES}

[1] S. T. Belinschi, M. Popa, and V. Vinnikov, On the operator-valued analogues of the semicircle, arcsine and Bernoulli laws, J. Operator Theory 70 (2013), 239-258. MR 3085826. DOI 10.7900/jot.2011jun24.1963.

[2] P. Biane, Processes with free increments, Math. Z. 227 (1998), 143-174. MR 1605393. DOI 10.1007/PL00004363.

[3] K. J. Dykema, Multilinear function series and transforms in free probability theory, Adv. Math. 258 (2007), 351-407. MR 2304321. DOI 10.1016/j.aim.2006.02.011.

[4] U. Franz, Monotone and Boolean convolutions for non-compactly supported probability measures, Indiana Univ. Math. J. 58 (2009), 1151-1185. MR 2541362. DOI 10.1512/iumj.2009.58.3578.

[5] T. Hasebe and H. Saigo, Joint cumulants for natural independence, Electron. Commun. Probab. 16 (2011), 491-506. MR 2836756. DOI 10.1214/ECP.v16-1647.

[6] - The monotone cumulants, Ann. Inst. Henri Poincaré Probab. Stat. 47 (2011), 1160-1170. MR 2884229. DOI 10.1214/10-AIHP379.

[7] F. Lehner, Cumulants in noncommutative probability theory, I: Noncommutative exchangeability systems, Math. Z. 248 (2004), 67-100. MR 2092722. DOI 10.1007/s00209-004-0653-0.

[8] N. Muraki, The five independences as natural products, Infin. Dimens. Anal. Quantum Probab. Relat. Top. 6 (2003), 337-371. MR 2016316.

DOI 10.1142/S0219025703001365.

[9] - Monotonic convolution and monotonic Lévy-Hinčin formula, preprint, 2000.

[10] M. Popa, A combinatorial approach to monotonic independence over a $C^{*}$-algebra, Pacific J. Math. 237 (2008), 299-325. MR 2421124. DOI 10.2140/pjm.2008.237.299.

[11] _ A new proof for the multiplicative property of the Boolean cumulants with applications to the operator-valued case, Colloq. Math. 117 (2009), 81-93. MR 2539549. DOI 10.4064/cm117-1-5. 
[12] M. Skeide, "Independence and product systems" in Recent Developments in Stochastic Analysis and Related Topics, World Scientific, Hackensack, N.J., 2004, 420-438. MR 2200526.

[13] R. Speicher, Combinatorial theory of the free product with amalgamation and operator-valued free probability theory, Mem. Amer. Math. Soc. 132 (1998), no. 627. MR 1407898. DOI 10.1090/memo/0627.

[14] R. Speicher and R. Woroudi, "Boolean convolution" in Free Probability Theory (Waterloo, Ontario, 1995), Fields Inst. Commun. 12, Amer. Math. Soc., 1997, 267280. MR 1426845.

[15] D. Voiculescu, "Symmetries of some reduced free product $C^{*}$-algebras" in Operator Algebras and Their Connections with Topology and Ergodic Theory (Busteni, 1983), Lecture Notes in Math. 1132, Springer, Berlin, 1985, 556-588. MR 0799593. DOI 10.1007/BFb0074909.

[16] - "Operations on certain non-commutative operator-valued random variables" in Recent Advances in Operator Algebras (Orléans, 1992), Astérisque 232, Soc. Math. France, Paris, 1995, 243-275. MR 1372537.

Takahiro Hasebe

Graduate School of Science

Kyoto University

Kyoto 606-8502

Japan

Current: Department of Mathematics

Hokkaido University

Kita-ku

Sapporo 060-0810

Japan

thasebe@math.sci.hokudai

Hayato Saigo

Nagahama Institute of Bio-Science and Technology

Nagahama 526-0829

Japan

h_saigoh@nagahama-i-bio.ac.jp 